\title{
Cusp Device
}

National Cancer Institute

\section{Source}

National Cancer Institute. Cusp Device. NCI Thesaurus. Code C49904.

A device consisting of flexible biocompatible membranes, implantable in the cardio-

vascular system, designed to prevent retrograde flow of blood but not impede a forward flow. 